\title{
A silence that speaks volumes
}

\author{
A biological gagging order, used in the fight against viruses, could \\ revolutionize our understanding of genetics and development. Trisha Gura \\ listens in on the world of gene silencing.
}

magine three researchers locked in a dark room with an elephant. One, grasping the trunk, decides it is a fire hose. The second, holding a leg, is sure it is a tree. The third, feeling the contours of an ear, imagines it is an uncooked pie crust. Then someone flicks the light switch, and all is revealed.

That, more or less, has been the story of one of the hottest new areas in biology post-transcriptional gene silencing (PTGS). Originally called 'co-suppression' by plant biologists, 'RNA interference' by those studying worms and flies, and 'quelling' by researchers working with fungi, scientists stumbled across PTGS independently as they struggled to work out why their experiments failed to go as planned. "One of the amazing things about this to me is how many discoveries have come up by accident," says David Baulcombe, a plant molecular biologist at the John Innes Centre in Norwich.

Now, researchers are recognizing that these seemingly disparate processes are intimately related. And with this realization has come a rush of excitement. By exploiting PTGS, biologists can switch off specific genes in a variety of organisms, allowing them to deduce the genes' functions. In addition, it can be used to alter traits, for example by slowing the production of a protein that causes fruit to ripen.

By silencing genes at different stages in an organism's embryonic growth, PTGS should also help to unravel the complexities of development. "We have this great new tool that cannot be overestimated," says Greg Hannon, a molecular biologist at the Cold Spring Harbor Laboratory on Long Island, New York.

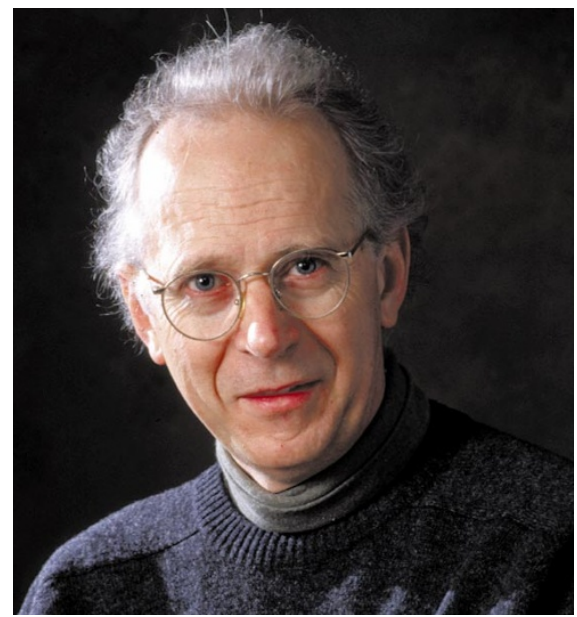

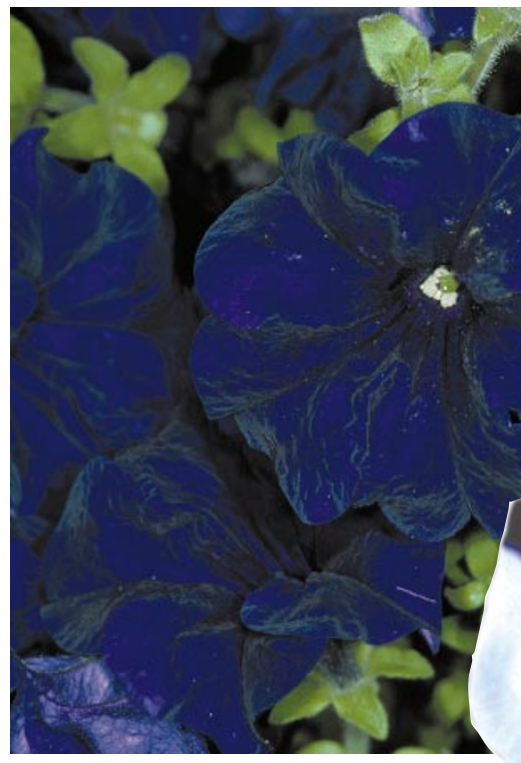

A lighter shade of failure? Attempts to deepen the purple hue of petunias by genetic modification produced unexpected results. Rather than heightening pigmentation, an inserted gene switched colour production off, creating variegated blooms (inset).

PTGS is now thought to be an ancient self-defence mechanism evolved to combat infection by viruses and transposons - parasitic stretches of DNA that can hop into an organism's genome, sometimes disrupting important genes. The response seems to be triggered by the presence in the host's cells of double-stranded RNA, or some other aberrant nucleic acid, which is indica-

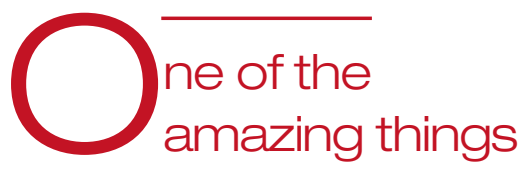
about this to me is how many discoveries have come up by accident

\section{David Baulcombe.}

tive of a viral assault. The RNA moving freely around a cell should normally be singlestranded messenger RNA (mRNA) - the intermediate between host genes and the proteins they encode. But when rogue RNA invades, the response is sharp and swift: any mRNAs matching the triggering nucleic acid's sequence are rapidly put out of action, effectively shutting down their parent gene. But PTGS is not discriminating. If the trigger mimics part of the host's genetic sequence, both host and viral genes are silenced.

\section{Flower power}

In plants, the story began with a quest for purpler petunias. More than a decade ago, a team led by molecular biologist Rich Jorgensen, then at DNA Plant Technology in Oakland, California, was trying to deepen the flowers' hues. Jorgensen's strategy hinged on boosting the activity of the gene for chalcone synthase, an enzyme involved 
in production of the anthocyanin pigments. The researchers hooked up the gene to a powerful promoter sequence and ferried this genetic construct into their petunias. However, instead of deep purple, many of the flowers grew up variegated, or virgin white ${ }^{1}$."We thought we must have made the construct wrong," recalls Jorgensen, now at the University of Arizona in Tucson.

On closer inspection, the researchers found that the revved-up chalcone synthase gene had somehow muted both itself and the normal petunia gene. Jorgensen coined the phrase 'co-suppression' to describe this paradoxical phenomenon. As he studied the process further, he noted that the white colour could be passed on to the next generation. However, some of the flowers partially reverted back to purple. This led him to suppose that co-suppression is mediated by a nucleic acid, presumably RNA, that had not taken up permanent residence in the petunia genome.

Most researchers dismissed the finding as a quirk of petunias, until two groups started to obtain similar results while working on plant RNA viruses. In Norwich, Baulcombe's team was expressing genes from the potato virus $\mathrm{X}$ in tobacco plants. The researchers hoped that viral proteins produced by the genes would stimulate the tobacco plants' defences, allowing the plants to resist subsequent attack by the virus itself. In many cases, this worked - but, contrary to prevailing theories of plant immunity, the introduced genes did not need to be translated into proteins to confer resistance. Indeed, the plants with the strongest resistance were those in which the introduced gene was silent ${ }^{2}$. Baulcombe eventually concluded that the introduced gene was co-suppressing both itself and the same gene in the virus. John Lindbo, William Dougherty and their colleagues at Oregon State University in Corvallis, working with the tobacco etch virus, obtained similar results ${ }^{3}$.

Both groups began to suspect that co-suppression had evolved to protect plants from viral attack. The case was strengthened when Baulcombe's team plus two other groups showed independently that several plant viruses produce proteins that stifle co-suppression $^{4-6}$. This suggested a classic evolutionary arms race between host and pathogen.

\section{Stop making sense}

As plant researchers began to understand the evolutionary significance of co-suppression, scientists working on the nematode worm Caenorhabditis elegans were obtaining strange results with a technique involving 'antisense' RNA. The theory behind antisense is simple: if you inject an organism with an RNA sequence that is 'complementary' to a particular mRNA, the two should zip up together into a doublestranded molecule, blocking production of the protein encoded by the mRNA.

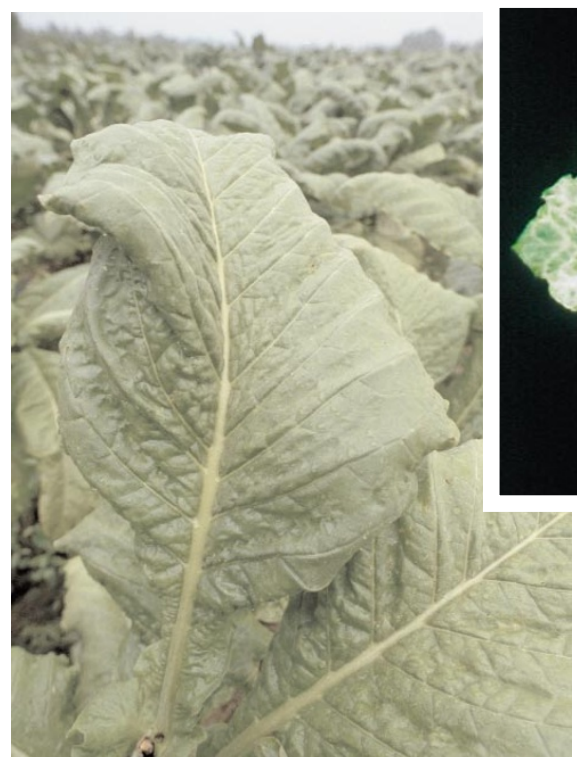

In 1995, Su Guo, a graduate student working in the lab of Kenneth Kemphues at Cornell University in Ithaca, New York, used antisense to disable a gene called par-1, which controls symmetry in C. elegans embryos ${ }^{7}$. But her results contained a strange finding: in many of Guo's control experiments - in which she injected 'sense' RNA, duplicating the target mRNA rather than complementing it - gene shut-down also took place.

It was not until 1998 that researchers led by Andrew Fire of the Carnegie Institution of Washington in Baltimore, Maryland, and Craig Mello of the University of Massachusetts Medical School in Cambridge figured out what was happening. Although preparations of single-stranded antisense RNA can shut down protein production by a particular gene, Fire and Mello found that doublestranded RNA works much better ${ }^{8}$. They

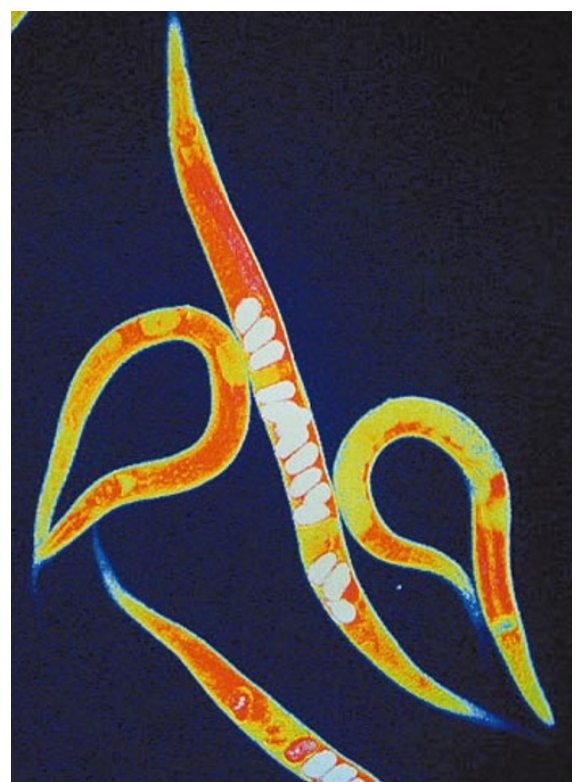

Silent witness: RNA interference is revealing the functions of genes in Caenorhabditis elegans. dubbed the phenomenon 'RNA interference' (RNAi). Previous demonstrations of gene suppression using antisense or sense RNA, Fire and Mello concluded, may have actually been caused by double-stranded molecules contaminating these preparations.

Under normal circumstances, doublestranded RNA should not exist in a worm's cells, unless an RNA virus or a transposon is in the process of copying itself - which again suggests that gene silencing is an antiviral mechanism. Indeed, Fire describes doublestranded RNA as "an Achilles heel" on which to focus attacks against RNA viruses.

In fungi, gene silencing was discovered during attempts to boost the production of an orange pigment by the mould Neurospora crassa. Giuseppe Macino and Carlo Cogoni at La Sapienza University in Rome introduced extra copies of a gene involved in

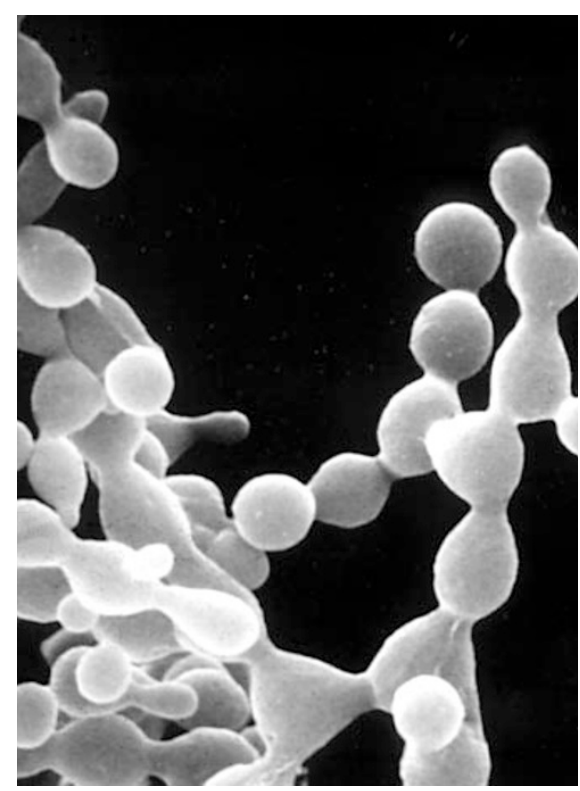

Breaking the mould: 'quelling' in Neurospora crassa shares genetic links with RNA interference. 
making a carotenoid pigment. But in around a third of their experiments, the engineered mould bleached out, rather than turning a deeper orange - something had suppressed the pigment gene'.

Other researchers had noticed similar gene-silencing effects, but found that the fungi often reverted back to normal. They wrote it off as an experimental artefact and never published their findings. But Macino and Cogoni were intrigued, called the phenomenon 'quelling', and tracked down a stable quelling strain that stayed white through multiple generations. By introducing further mutations and selecting those that caused the fungus to revert back to orange, Macino and Cogoni identified a suite of genes, including qde-1, qde-2 and qde-3, crucial to the quelling process ${ }^{10}$.

The discovery of these genes, and of similar genes involved in various forms of PTGS in other organisms, has been central to the growing realization that these processes are fundamentally linked - although the details of the mechanisms involved are still uncertain (see 'How does it work?', page 807). Intriguingly, qde-2 shares much of its sequence with a gene needed for RNAi in C. elegans ${ }^{11}$.

Other observations have strengthened the idea of different variants of gene silencing sharing a common mechanism with deep evolutionary roots. In particular, both $C$.

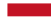 \\ $\mathrm{T}$ here is growing excitement over gene silencing, which promises to be a favourite tool of biologists for years to come.}

elegans and the fruitfly Drosophila exhibit a gene-silencing phenomenon comparable to that seen in plants, in addition to $\mathrm{RNAi}^{12,13}$. This co-suppression is triggered by the expression of a second copy of a normal fly or worm gene, rather than the presence of double-stranded RNA. And last month, René Ketting and Ronald Plasterk of the Netherlands Cancer Institute in Amsterdam revealed four genes that are required for both RNAi and cosuppression in C. elegans $^{14}$. Genes related to them seem to be involved in one process or the other, but not both.

But for many biologists, details of the mechanisms of PTGS, and the links between its different forms, are of secondary interest to its potential as a research tool. For develop-

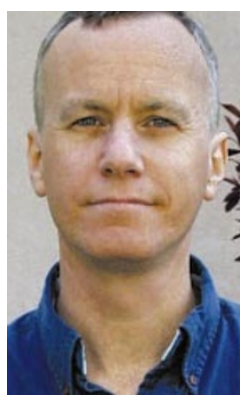

mental studies, RNAi offers strong advantages over competing geneknockout techniques such as disrupting a gene by placing a marker sequence in the middle of its coding region because it can be applied selectively at different developmental stages. Carthew: pioneered Often, eliminating a gene gene silencing in kills the embryo before fruitflies. anything can be determined about what the gene might do. Even if the embryo survives, abnormalities introduced early in development can mask subtle secondary functions that a gene may perform at later stages.

\section{A valuable tool}

In C. elegans, RNAi is already yielding an impressive harvest of discoveries. In the past few years, a variety of researchers have used RNAi to investigate the functions of a host of genes involved in cell division ${ }^{15-19}$. RNAi can be triggered simply by injecting adult worms with double-stranded RNA, soaking the animals in the nucleic acid, or by engineering Escherichia coli to produce the appropriate two-stranded RNA and feeding the bacteria to the worms. 


\section{How does it work?}

Although the mechanisms of gene silencing are far from completely understood, the working hypothesis goes like this: the initial trigger is the presence in the host's cells of an aberrant RNA. This could be a double-stranded RNA, a shortened RNA that lacks its 'cap' or 'tail', or a conventional RNA that is present in unusually large quantities - all of which can indicate that a virus is on the attack.

The host organism's response is to call on enzymes that slice and dice the offending RNA into pieces around 25 nucleotides long. At some stage - either before or after the formation of these fragments - the rogue RNA is copied many times over, to amplify the alarm signal. The fragments then spread throughout the host. Antisense strands, complementary to the target mRNA, bind to the target and prompt other enzymes to disable it.

Several recent discoveries have contributed to this hypothesis. The sequence of the qde- 1 gene, for instance, involved in gene silencing in fungi, suggests that it encodes an enzyme belonging to a family called RNA-dependent RNA polymerases ${ }^{24}$. This enzyme may amplify the rogue RNA.

The fact that the triggering RNA is cut into small fragments was revealed by work in plants and Drosophila cell cultures. Last year, David Baulcombe's group at the John Innes Centre in Norwich found that transgenic tobacco plants undergoing co-suppression induced by potato $X$ virus make 25-nucleotide-long fragments that correspond to the target of gene silencing ${ }^{25}$.

Meanwhile, Greg Hannon of the Cold Spring Harbor Laboratory on Long Island, New York, has been inducing RNAi in Drosophila cells, pulverizing them, and then screening different biochemical extracts for gene-silencing activity.

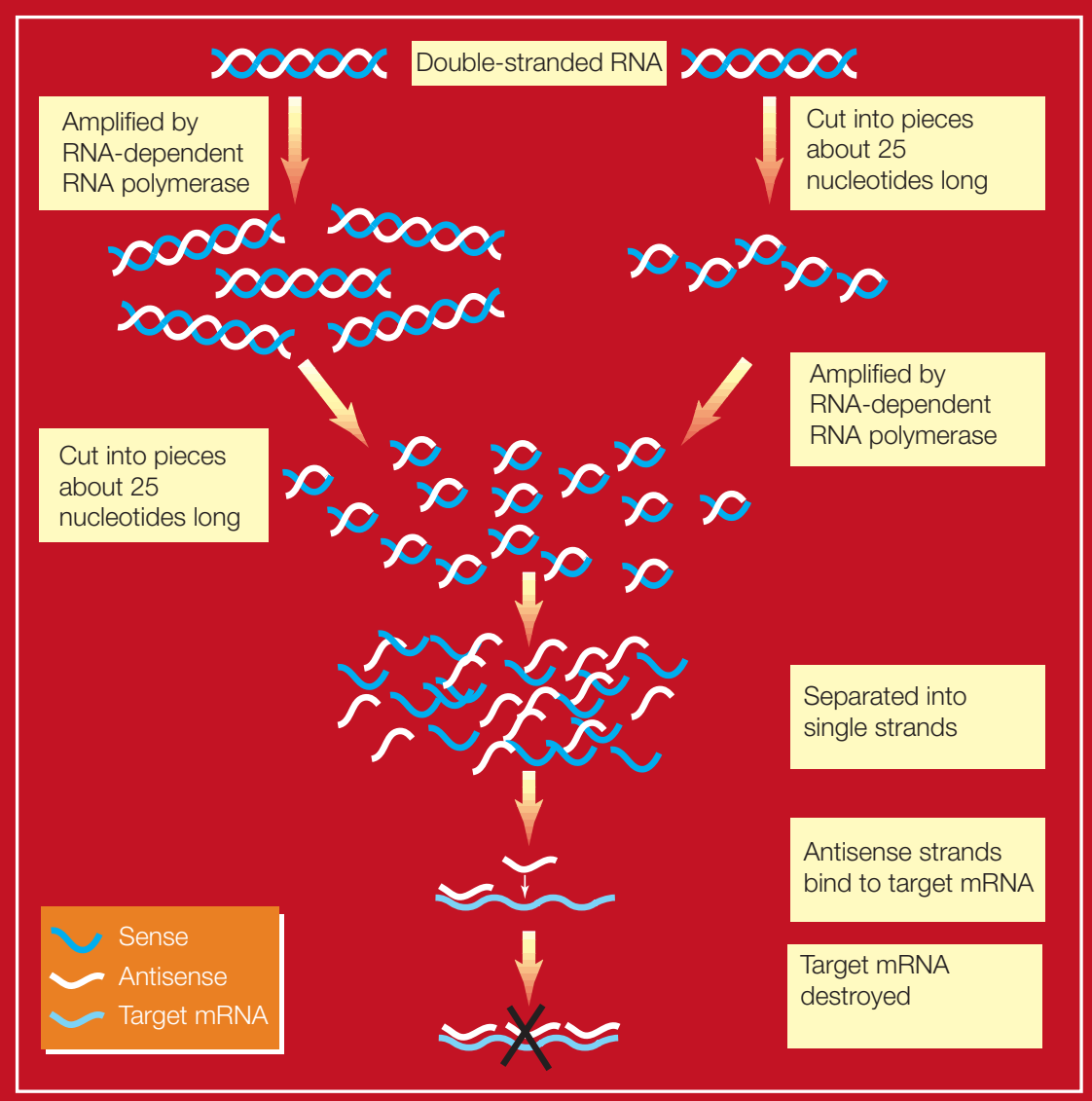

The active fractions again contained discrete RNAs, around $\mathbf{2 5}$ nucleotides long, corresponding to sequences from the silenced gene ${ }^{26}$.

Most recently, Phillip Zamore of the University of Massachusetts Medical School in Worcester and system, have shown that these RNA fragments are between 21 and 23 nucleotides long. These seem to spell doom for the target mRNA by directing enzymes to cut it into pieces ${ }^{27}$. his colleagues, working with a similar in vitro
In Drosophila, however, engineering yeast cells to make double-stranded RNA and feeding this yeast to the flies failed to work. But Richard Carthew and his colleagues at the University of Pittsburgh have solved the problem. They microinject Drosophila embryos with double-stranded RNA, or shoot the molecules into the flies with a 'gene gun' - a mechanical device that propels nucleic acids into cells under high pressure. Carthew's team has also developed a technique of engineering flies to carry a stretch of DNA containing an inverted repeat of the gene to be silenced. The flies' DNA presumably transcribes the repeat, forming an RNA molecule that folds back into a hairpin-like structure - in essence, a double-stranded RNA. The Pittsburgh group has already used RNAi to silence at least 20 fruitfly genes, including frizzled 2 and wingless, which are involved in wing development ${ }^{20}$.

Meanwhile, Hannon's group at Cold Spring Harbor is hoping to gain insights into cancer biology by using RNAi in Drosophila cell cultures to look at genes, such as cyclin $E$ and $m y c$, that regulate the cycle of cell growth and division. Knocking out cell-cycle genes can yield ambiguous results, says Hannon, because the genes often have relatives that can mimic their function, picking up the slack. With RNAi, it is possible to knock out several related genes at once. "What distinguishes this system is that is it flexible," enthuses Hannon.

The use of RNAi is set to take off in both C. elegans and Drosophila, as the near-complete genome sequence of each organism has now been published - the worm in 1998 , the fly last month. Both genomes contain a wealth of genes whose functions are waiting to be discovered.

In plants, co-suppression techniques have already gained widespread use. Guy della-Cioppa and his colleagues at Biosource Technologies in Vacaville, California, are using co-suppression in an attempt to uncover the function of orphan genes in Arabidopsis thaliana, the leading model organism in plant biology. Using the tobacco mosaic virus to shuttle in thousands of different genetic sequences, the Biosource researchers are knocking out genes systemat-

$\approx$ ๑ 2000 Macmillan Magazines Ltd

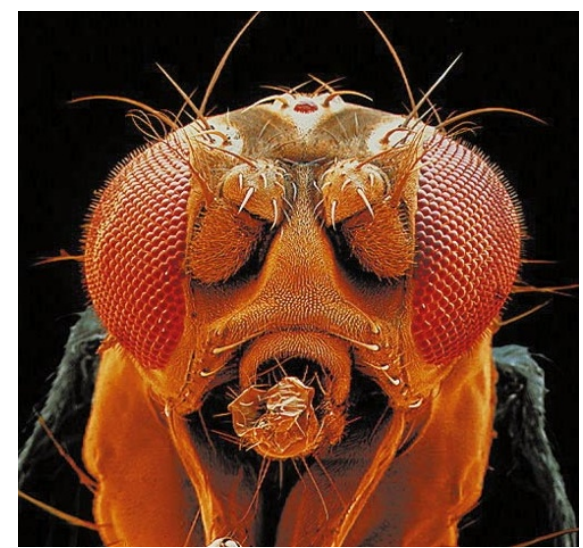

Functional fit: gene silencing is creating a buzz among researchers of the fruitfly's genome.

ically and screening for desirable traits such as disease resistance or drought tolerance.

In addition to its use as a laboratory tool, co-suppression is also being used commercially. Neal Gutterson and his colleagues at DNA Plant Technology have used it to alter floral patterns in petunias and knocked out a 
hormone in carnations to allow the flowers to last longer. Researchers at AstraZeneca's laboratory in Norwich, meanwhile, have used co-suppression to silence the gene in tomatoes for the enzyme polygalacturonase, which digests cell walls as fruit ripens. The resulting tomatoes, which are used to make purée, can be picked later in the season. As a result, they develop a stronger flavour.

\section{Added backbone}

There are now suggestions that PTGS may also work in vertebrates. This has surprised many researchers, because these 'higher' animals were thought to have evolved more powerful responses to RNA viruses and transposons. In particular, mammals possess an enzyme called protein kinase $\mathrm{R}$, which shuts down a cell's protein-synthesis machinery in response to the presence of double-stranded RNA, sometimes provoking cell suicide. Indeed, attempts to induce RNAi in cultures of mammalian cells or in whole animals have met with failure.

However, it now seems that RNAi may operate in vertebrates at the earliest stages of development. In February, Magdalena Zernicka-Goetz at the University of Cambridge reported success in early-stage mouse embryos $^{21}$. She injected double-stranded RNA into mouse egg cells, or into embryos at the 8- or 16-cell stages, and was able to block the expression of genes that help determine polarity - the clustering of cells at an embryo's head and tail that helps assign its eventual symmetry. "The potential is there to have it work in mammals," says Hannon.

Similar results are emerging from work on zebrafish, another popular organism for studies of vertebrate developmental biology. Although the efficiency of gene silencing is much lower than that reported in ZernickaGoetz's mouse experiments, and effects other than RNAi may also be involved, Anders Fjose and his colleagues at the University of Bergen in Norway microinjected double-stranded RNA for three genes of known function into zebrafish embryos and witnessed the same developmental defects that are seen if the genes are mutated ${ }^{22}$. Researchers led by Margaret Kirby and Yin Xiong Li at the Medical College of Georgia in Augusta have since obtained similar results ${ }^{23}$.

As more researchers focus on PTGS, new discoveries are emerging almost by the week. "There are plenty of mysteries still," says Hannon. But as understanding of gene silencing improves, those mysteries are fast being outshone by a sense of growing excitement over a technique that promises to be a favourite tool of biologists for years to come.

Trisha Gura is a freelance writer in Cleveland, Ohio.

1. Napoli, C., Lemieux, C. \& Jorgensen, R. Plant Cell 2, 279-289 (1990).
2. Angell, S. M. \& Baulcombe, D. C. EMBO J. 16, 3675-3684 (1997)

3. Dougherty, W. G. et al. Mol. Plant-Microbe Interact. 7, 544-552 (1994).

4. Anandalakshmi, R. et al. Proc. Natl Acad. Sci. USA 95, 13079-13084 (1998)

5. Kasschau, K. D. \& Carrington, J. C. Cell 95, 461-470 (1998),

6. Brigneti, G. et al. EMBO J. 17, 6739-6746 (1998).

7. Guo, S. \& Kemphues, K. J. Cell 81, 611-620 (1995).

8. Fire, A. et al. Nature 391, 806-811 (1998).

9. Cogoni, C., Romano, N. \& Macino, G. Antonie Van Leeuwenhoek 65, 205-209 (1994).

10. Cogoni, C. \& Macino, G. Proc. Natl Acad. Sci. USA 94, 10233-10238 (1997)

11. Catalanotto, C., Azzalin, G., Macino, G. \& Cogoni, C. Nature 404, 245 (2000).

12. Pal-Bhadra, M., Bhadra, U. \& Birchler, J. A. Cell 90, 383-387 (1997).

13. Jones, A. R. \& Schedl, T. Genes Dev. 9, 1491-1504 (1995).

14. Ketting, R. F. \& Plasterk, R. H. A. Nature 404, 296-298 (2000).

15. Schumacher, J. M., Golden, A. \& Donovan, P. J. J. Cell Biol. 143, 1635-1646 (1998).

16. Gonczy, P., Pichler, S., Kirkham, M. \& Hyman, A. A. J. Cell Biol. 147, 135-150 (1999).

17. Moore, L. L., Morrison, M. \& Roth, M. B. J. Cell Biol. 147, 471-480 (1999).

18. Jantsch-Plunger, V. \& Glotzer, M. Curr. Biol. 9, 738-745 (1999). 19. Chase, D. et al. Genesis 26, 26-41 (2000).

20. Kennerdell, J. R. \& Carthew, R. W. Cell 95, 1017-1026 (1998).

21. Wiany, F. \& Zernicka-Goetz, M. Nature Cell Biol. 2, 70-75 (2000).

22. Wargelius, A., Ellingsen, S. \& Fjose, A. Biochem. Biophys. Res. Commun. 263, 156-161 (1999).

23. Li, Y. X. et al. Dev. Biol. 217, 394-405 (2000).

24. Cogoni C. \& Macino G. Nature 399, 166-169 (1999).

25. Hamilton, A. J. \& Baulcombe, D. C. Science 286, 950-952 (1999).

26. Hammond, S. M., Bernstein, E., Beach, D. \& Hannon, G. J. Nature 404, 293-296 (2000).

27.Zamore, P. D., Tuschi, T., Sharp, P. A. \& Bartel, D. P. Cell 101, 25-33 (2000). 